\title{
Alternatives Initiative in Sri Lanka: Pre- and Post-Conference Workshops at the Inaugural Scientific Conference of the Sri Lanka Association for Laboratory Animal Science
}

\author{
Mangala Gunatilake ${ }^{1}$, Francois Busquet ${ }^{2}$, and Mohammad A. Akbarsha ${ }^{3}$ \\ ${ }^{1}$ Department of Physiology, Faculty of Medicine, University of Colombo, Sri Lanka; ${ }^{2}$ Center for Alternatives to Animal \\ Testing (CAAT)-Europe, Konstanz, Germany; ${ }^{3}$ Mahatma Gandhi-Doerenkamp Center (MGDC) for Alternatives, Bharathidasan \\ University, Tiruchirappalli, India
}

http://dx.doi.org/10.14573/altex.1403121

\section{Introduction}

Ethical principles to reduce pain and suffering in animals during scientific experiments made scientists understand the im portance of alternative methods to implement the 3Rs concept, which later led to the development and validation of different alternative models. European countries are far ahead in this venture compared to most Asian countries. Therefore, a PreConference (January 24, 2014) and a Post-Conference (January 26,2014 ) workshop were conducted on several alternative models during the Inaugural Scientific Conference of the Sri Lanka Association for Laboratory Animal Science held on January 25,2014 , as an initiative to introduce alternatives to ani- mal experiments to the Sri Lankan scientific community. This was possible through the contacts developed by Prof Mangala Gunatilake, who spearheaded the establishment of the Sri Lanka Association for Laboratory Animal Science (SLALAS) in December 2012, with international scientists concerned with animal welfare and alternatives. In her introductory remarks, she gave a brief overview of the concept of alternatives and its relevance to Sri Lanka. Inaugurating the Post-Conference workshop, the Most Venerable Olande Ananda Thera, a Bud dhist Monk of High Order, explained the value of lives, whether they are human or animal, and the importance of respecting all life. This drove home the importance of looking for alternatives to animals in experiments and research. 


\section{Contribution from CAAT-Europe}

CAAT-Europe's involvement in SLALAS was to advocate alternatives to animal testing and to provide the necessary support and training on-site for a 3Rs test method at two universities (Uva Wellassa and Colombo). Zebrafish is a native species in Sri Lanka and is bred for ornamental purposes only but their use as a research model so far had not been considered. In Germany, zebrafish eggs are used for wastewater toxicity testing (ISO 15088, 2007) and at the OECD level for chemical toxicity testing (TG 236). Zebrafish are a low cost model, easy to culture compared to cells, and have multiple applications for academic purposes (Nagel, 2002). Toxicity testing on zebrafish embryos is considered a reduction and refinement method.

Careful preparation was undertaken to ensure the success of the method transfer: 1) compilation of a check-list of material and regular communication with the Sri Lankan faculties, 2) training of CAAT-Europe staff at the Merck KgaA Institute of Toxicology headed by Dr Huebler, and 3) provision of video material by Dr Schirmer's department (EAWAG) to illustrate the fish embryo tests in case no eggs were laid for use in the demonstration. The content of the training, as provided by Dr Francois Busquet, European Policy Coordinator of the Centre for Alternatives to Animal Testing (CAAT), covered zebrafish handling (food frequency, type of food, water quality parameters, etc.), egg production and collection, description of ZF embryological stages, chemical exposure (ethanol), microscope observation of lethal endpoints, $\mathrm{LC}_{50}$ calculation, and introduction to environmental hazard and risk assessment (PEC/PNEC). In a nutshell, the objectives achieved were the following: 1) establishment of a zebrafish laboratory unit and maintenance of egg production for research and toxicological studies in Uva Wellassa university, 2) training of researchers in the zebrafish embryo toxicity test for chemicals and waste water at both universities, and 3) sensitization to the use of zebrafish eggs as a toxicological model rather than mammalian models.

The successful training led to enthusiasm among the students and commitment from the faculty to investigate local environmental issues (e.g., linked with heavy metals) as well as indigenous drugs using zebrafish eggs.

\section{Contribution from MGDC (India) and DZF (Switzerland)}

The Mahatma Gandhi-Doerenkamp Center (India) and its sponsor organization, the Doerenkamp-Zbinden Foundation (Switzerland), contributed in two ways: 1) sensitization of the workshop participants about the problems (scientific as well as ethical) of animal testing of drugs, cosmetics, environmental chemicals/agrochemicals, food additives/preservatives/colorants, consumer products, etc. and 2) training in the recently developed in vitro model Integrated discrete Multiple Organ Coculture (IdMOC). Dr Mohammad A. Akbarsha, from MGDC, representing the two organizations, highlighted the need for alternative risk assessment strategies, deriving examples from drug development scenarios and the many failures following in vivo testing. He underscored the importance of in vitro and in silico approaches. Among the different approaches in in vitro toxicology and pharmacology, IdMOC provides advantages over the culture of a single cell type or a combination of two or more cell types in the same culture plate or well in that in IdMOC cells from different tissues are cultured separately in different wells contained in the same chamber of the same specially designed plate. For example, a 96-well IdMOC plate is divided into 16 chambers, which are totally separated from each other. The wells are so designed that different cells can be cultured separately in the 6 wells of every chamber. After culturing the cells (primary cells or established cell lines) separately in their respective media, an overlying universal medium that represents the blood, containing the toxicant/drug candidate, is added in such a way that wells are flooded and the medium rises to a level above the brim of the wells so as to provide a uniform environment for all six wells of the chamber. The different chambers can be used to test different concentrations of the test substance, different test substances at the same time, or different combinations of cells. The overlying universal medium can also be analyzed for metabolites so as to assess whether one or more target cells are dependent on the metabolites produced by an organ that metabolizes the chemical, say hepatocytes (Li et al., 2012). It was explained how this could bridge the gap between the use of a single type of cell/tissue culture and whole animals/humans. The In Vitro ADMET Labs, Columbia, MD, USA, kindly provided IdMOC plates for a demonstration of this technology as a gift.

\section{Contribution from SLALAS}

On the initiative of Dr Helena Kandarova, Executive Director, MatTek In Vitro Life Science Laboratories, USA, and relying on basic training undergone at the $18^{\text {th }}$ European Congress on Alternatives to Animal Testing (EUSAAT), Prof Gunatilake enlightened the audience about the In vitro EpiDerm skin irritation test, which is a validated alternative method for skin irritation testing that is accepted for regulatory chemical safety testing. She highlighted the limitations of extrapolating the findings from rabbit skin tests to humans due to the high sensitivity of rabbit skin compared to human skin. A mock demonstration of the model was later performed using material kindly provided by Dr Helena Kandarova at the workshop "Toxicity Testing for Industrial Purposes and Possible Alternatives" held on March 14, 2014.

\section{Conclusion}

The models explained/demonstrated at the Pre- and Post-Conference workshops on alternatives to animal testing were highly 
interactive and developed an interest in the participants to use possible alternatives with available facilities in order to reduce the use of animals in scientific experiments, the first ever initiative in this direction in the Sri Lankan scientific community.

\section{References}

Li, A. P., Uzgare, A., and LaForge, Y. S. (2012). Definition of metabolism-dependent xenobiotic toxicity with co-cultures of human hepatocytes and mouse 3T3 fibroblasts in the novel integrated discrete multiple organ co-culture (IdMOC) experimental system: results with model toxicants aflatoxin B1, cyclophosphamide and tamoxifen. Chem Biol Interact 199, 1-8. http://dx.doi.org/10.1016/j.cbi.2012.05.003
Nagel, R. (2002). DarT: The embryo test with the zebrafish (Danio rerio) - a general model in ecotoxicology and toxicology. ALTEX 19, 38-48. http://www.altex.ch/All-issues/Issue. $50 . \mathrm{html}$ ?iid $=68 \&$ aid $=9$

\section{Correspondence to}

M. A. Akbarsha, PhD

Mahatma Gandhi-Doerenkamp Center (MGDC)

for Alternatives to Use of Animals in Life Science Education Bharathidasan University

Tiruchirappalli 620024

India

Phone: +91 9790995854

e-mail:mgdcaua@yahoo.in 